Identity Processes and Well-Being: Exploring Different Types of Identity Exploration

\author{
Phuong Linh L. Nguyen
}

University of Minnesota

Author Note

This preprint has not been peer-reviewed. The current manuscript was completed as a course requirement and will be updated. Correspondence concerning this article should be address to Phuong Linh L. Nguyen, nguy4006@umn.edu 


\begin{abstract}
The identity exploration process includes exploration in-depth, exploration in-breadth, and ruminative exploration, which cover the process of examining multiple potential future plans and directions, investigating them deeply and sometimes ruminatively, before arriving at a committed identity. Each of the three exploration types have demonstrated different associations with various measures of well-being and ill-being in the literature, due to their unique approaches to identity development. The current study investigated the relationship between the two adaptive exploration processes - exploration in-depth and in-breadth - with measures of self-esteem and distress in a diverse US-based sample. We found that both in-depth and inbreadth exploration were positively related to self-esteem. Interestingly, when ruminative exploration was included in the models, thus partialling out the maladaptive component of indepth or in-breadth exploration, both of these adaptive processes showed a negative association with distress that were not previously present in individual simple linear models. Future research should explicitly control for the cross-correlation patterns among these constructs for a clearer picture of the consequences and correlates of the identity development process.
\end{abstract}

Keywords: identity development, identity exploration, self-esteem, distress 


\section{Identity Processes and Well-Being: Exploring Different Types of Identity Exploration}

Identity refers to people's sense of self and how they situate themselves in the world. Identity development was first proposed as a developmentally normative process, in which people resolve an identity crisis primarily during adolescent years (Erikson, 1968). Initial empirical investigations of identity mainly applied categorical completion statuses: moratorium, diffusion, foreclosure, and achievement (Marcia, 1966). In addition, two broad categories of identity confusion and coherence (or synthesis) are also used extensively (Rosenthal et al., 1981), and they directly examine the two extremes in Erikson's identity construct. Different identity stages are differentially related to various measures of well-being in emerging adults, including anxiety, depression, aggression, self-esteem, and life satisfaction, all in the theoretically congruent direction (Schwartz et al., 2011). That is, identity stages associated with high commitment and synthesis (i.e. achievement and foreclosure) were generally associated with well-being, whereas stages characterized by more uncertainty and exploration (i.e., moratorium and diffusion) were more highly associated with health-compromising behaviors.

Contemporary approaches to identity research expand beyond these linear, discrete categories to examine individual processes of exploration and commitment (Luyckx et al., 2006a, 2008). The exploration process, in which people actively search and examine different options, includes exploration in-breadth, exploration in-depth, and ruminative exploration. The commitment process, in which people decide on an integrated sense of self, includes commitment making, identification with commitment, and reconsideration of commitment. Multiple theoretical models have been proposed to organize the exploration and commitment processes to form different identity trajectories, including the dual-cycle model of exploration followed by commitment (Luyckx, 2006a; Meeus, 2011) and the certainty-uncertainty model of commitment - exploration in-depth - reconsideration of commitment in which people start with a rough identity concept, and not a blank slate, before further exploration (Crocetti et al., 2008). Different identity styles have also been proposed, including the informational, normative, and diffuse-avoidant styles (Berzonsky, 1989).

In examining the classical and contemporary approaches to identity development, it is clear that the exploration process not only plays a crucial role but is also a generic description for what is a varied group of processes. In fact, the three exploration processes - in-breadth, in- 
depth, and ruminative - have shown differential associations with various personality and wellbeing measures. Exploration in-breadth involves considering multiple options in the investigation process before arriving at a suitable option for commitment. Exploration in-depth refers to the concentrated and thorough investigation of one identity option (Luyckx, et al., 2006a). These two dimensions are distinctive in their approach and in fact have different determinants and correlates. Exploration in-depth is positively associated with adjustment and close parental relationships, whereas exploration in-breadth is associated with mal-adjustment, familial independence, and low Conscientiousness (Luyckx et al., 2006a, 2006b). Nonetheless, they are both characteristic of the normative exploration processes: weighing between different options and seriously considering few potential candidates, and they are both positively correlated with personality traits openness and curiosity (Crocetti et al., 2008, Luyckx et al., 2006b). On the other hand, ruminative exploration was proposed as the maladaptive side of identity exploration, which is characterized by heightened anxiety and self-rumination (Luyckx et al., 2008). Taken together, these three facets constitute a broad exploration domain and explained the mixed associations of exploration with both well- and ill-being.

\section{Current paper}

The current paper examined the differential relationships between different types of identity exploration and measures of well- and ill-being. In particular, we hypothesized that H1a) Exploration in-depth would positively correlate with self-esteem and negatively with distress, and $\mathrm{H} 1 \mathrm{~b}$ ) this effect would remain after including ruminative exploration in the model. Secondly, we hypothesized that $\mathrm{H} 2 \mathrm{a}$ ) Exploration in-breadth would negatively correlate with self-esteem and positively with distress, and $\mathrm{H} 2 \mathrm{~b}$ ) this effect would disappear after including ruminative exploration in the model. In other words, we aimed to establish that whereas the positive implications of exploration in-depth were independent of ruminative exploration, any negative implications of exploration in-breadth would be accounted for by ruminative exploration. That is, exploration in-breadth in of itself would not be associated with ill-being.

\section{Method}

\section{Participants}


Data were collected in a diverse national sample of 1,260 US-based participants aged 1830 years old $(M=24.41, S D=3.87)$. The sample was evenly split into three racial/ethnic categories by design: White, Black, or Latino. The majority of participants identified as female (72.4\%) and 24.7\% identified as male. Participants were recruited by Qualtrics survey panels and completed a one-time survey on the Qualtrics platform. ${ }^{1}$ Data collection included various additional measures that were not included in the current study.

\section{Measures}

Identity. The Dimensions of Identity Development Scale (Luyckx et al., 2008) included 25 items evenly distributed to measure five separate identity processes: exploration in-depth, exploration in-breadth, ruminative exploration, commitment making, and identification with commitment. The current study used only the three measures of identity explorations in our analyses. These subscales were relatively reliable, with Cronbach's alphas of 0.70 (in-depth), 0.73 (ruminative), and 0.80 (in-breadth). Interestingly, all three variables were negativelyskewed, with an average of 3.89, 3.62, and 3.52 for in-breath, in-depth, and ruminative exploration, respectively, on a Likert scale of one to five. This suggests that the sample indicates slightly higher endorsement of different exploration categories, both adaptive and maladaptive ones, compared to the scale average. Norms for scale scores were not reported for the original scale development documentation (Luyckx et al., 2008), but compared to a sample of 437 Greek adolescents $\left(M_{\text {age }}=15.7\right.$; Mastrotheodoros \& Motti-Stefanidi, 2017), this is consistent for inbreadth and in-depth dimensions. However, this past paper reported much lower than average scores for ruminative exploration (2.44 out of 5). For ease of presentation, in-depth and inbreadth exploration are henceforth referred to as adaptive exploration to contrast with ruminative exploration, which is a maladaptive exploration dimension.

Self-esteem. The Rosenberg Self-Esteem Scale (Rosenberg, 1965) included ten items to measure positive and negative self-esteem on a four-point Likert scale. The negative items were reverse-scored for an aggregate self-esteem variable. The measure was reliable, with Cronbach's

\footnotetext{
${ }^{1}$ For a detailed description of the demographic variables and scale items, please refer to the codebook at https://rpubs.com/nguyenllpsych/identity5960
} 
alpha of 0.87. For example, positively worded items included "On the whole, I am satisfied with myself” and negatively worded items included "At times, I think I am no good at all."

Distress. The Kessler Psychological Distress Scale (Kessler et al., 2002) included ten items to measure a general distress variable on a five-point Likert scale. All items asked participants to indicate the frequency of experiencing different distressing emotions over the past month, such as feeling hopeless or restless and fidgety. The measure showed high internal consistency with Cronbach's alpha of 0.93 .

\section{Data analytic plan}

With an alpha of 0.05 , we had $80 \%$ power to detect a correlation of 0.08 and $99.99 \%$ power to detect a correlation of 0.2. The middle third of effects in psychology ranges from .2 to .3 (Hemphill, 2003). Thus, we believe the sample to be well-powered for the current analyses.

For each of the two main research questions, we conducted a two-stage process: First, simple linear regression models were fitted to examine individual relationships between adaptive exploration dimensions and self-esteem and distress. Second, an additional predictor of ruminative exploration was added to examine any change to the previous relationship. If any significant associations were negated after including the additional term, we may infer that ruminative exploration accounted for those associations. To the contrary, if the associations remained, we may infer that adaptive exploration dimensions had a significant association with well- and ill-being independently of their relationship with ruminative exploration. All analyses were preregistered after data collection was completed and conducted in R (R Core Team, 2020). The preregistration record and analysis script are available on the OSF repository ${ }^{2}$.

\section{Results}

\section{Descriptives}

We observed a significant positive correlation between the two adaptive exploration dimensions $(r=.66, p<.001)$. In addition, ruminative exploration positively correlated with both exploration in-breadth $(r=.42, p<.001)$ and exploration in-depth $(r=.21, p<.001)$. This indicated a global exploration factor that contributes to both adaptive and maladaptive

\footnotetext{
${ }^{2}$ https://osf.io/dfgzy/
} 
dimensions. In addition, bivariate correlations between the three exploration dimensions and distress and self-esteem are reported in Table 1. These relationships are discussed further in the next sections.

Table 1. Bivariate correlations among identity exploration dimensions, distress, and self-esteem.

\begin{tabular}{|c|c|c|c|c|c|}
\hline Variable & $M(S D)$ & $\overline{\mathrm{BE}}$ & $\overline{\mathrm{DE}}$ & RE & KD \\
\hline Exploration in-depth & $3.89(.73)$ & & & & \\
\hline Exploration in-depth & $3.62(.74)$ & $\begin{array}{c}.66 * * * \\
{[.63, .69]}\end{array}$ & & & \\
\hline Ruminative exploration & $3.52(.82)$ & $\begin{array}{c}.42 * * * \\
{[.37, .46]}\end{array}$ & $\begin{array}{c}.21 * * * \\
{[.15, .26]}\end{array}$ & & \\
\hline Distress & $2.72(1.01)$ & $\begin{array}{c}.11 * * * \\
{[.06, .17]}\end{array}$ & $\begin{array}{c}.03 \\
{[-.03, .08]}\end{array}$ & $\begin{array}{c}.45^{* *} \\
{[.41, .50]}\end{array}$ & \\
\hline Self-esteem & $2.78(.61)$ & $\begin{array}{c}.12 * * * \\
{[.07, .17]}\end{array}$ & $\begin{array}{c}.26 * * * \\
{[.21, .31]}\end{array}$ & $\begin{array}{c}-.38 * * * \\
{[-.43,-.33]}\end{array}$ & $\begin{array}{c}-.60 * * * \\
{[-.64,-.57]}\end{array}$ \\
\hline
\end{tabular}

Note. $M=$ mean, $S D=$ standard deviation, $\mathrm{BE}=$ exploration in-breadth, $\mathrm{DE}=$ exploration indepth, $\mathrm{RE}=$ ruminative exploration, $\mathrm{KD}=$ distress, $\mathrm{RSE}=$ self-esteem. Values in square brackets indicate the $95 \%$ confidence interval for each correlation. $* * *$ indicates $p<.001$

\section{Exploration in-depth}

Simple linear regression showed a positive relationship between exploration in-depth and self-esteem $(r=.26, B=.22, p<.001)$. As participants indicated a higher level of thorough and thoughtful future planning, they also expressed a higher degree of positive self-attitudes. As hypothesized, this relationship remained when ruminative exploration was added as a predictor, which showed a negative association with self-esteem in the multiple regression model $(B=-$ $.339, p<.001)$. This indicated that the positive association between exploration in-depth and self-esteem was not accounted for by ruminative exploration. 
Table 2. Multiple regression results for self-esteem with in-depth and ruminative exploration

\begin{tabular}{ccccc}
\hline \hline & Estimate & Std. Error & $t$-value & $p$-value \\
\hline Intercept (Self-esteem) & 2.915 & .090 & 32.34 & $<.001$ \\
Exploration in-depth & .291 & .021 & 14.13 & $<.001$ \\
Ruminative exploration & -.339 & .019 & -18.13 & $<.001$ \\
\hline
\end{tabular}

Contrary to our hypotheses, there was no association between exploration in-depth and distress $(r=.03, B=.04, p=.29)$. Because this main effect was not significant, we were technically no longer interested in whether ruminative exploration would change the relationship. However, interestingly, multiple regression results showed a significant association for both exploration in-depth $(B=-.10, p=.007)$ and ruminative exploration $(B=.58, p<.001)$. This indicated that when holding the other variable constant, distress decreased as exploration indepth increase and distress increased as ruminative exploration increased.

Table 3. Multiple regression results for distress with in-depth and ruminative exploration

\begin{tabular}{ccccc}
\hline \hline & Estimate & Std. Error & $t$-value & $p$-value \\
\hline Intercept (Distress) & 1.013 & .153 & 6.632 & $<.001$ \\
Exploration in-depth & -.095 & .035 & -2.690 & .007 \\
Ruminative exploration & .580 & .032 & 18.224 & $<.001$ \\
\hline
\end{tabular}

\section{Exploration in-breadth}

Simple linear regression showed a positive relationship between exploration in-breadth and self-esteem $(r=.12, B=.10, p<.001)$. Contrary to our hypotheses, as participants indicated higher frequency of thinking about future plans and directions, they also expressed more positive self-attitudes. This relationship remained after including ruminative exploration as a predictor, which was negatively related to self-esteem in the multiple regression model $(B=-.39, p<.001)$. This indicated that the positive association between exploration in-breadth and self-esteem was not accounted for by ruminative exploration. 
Table 4. Multiple regression results for self-esteem with in-breadth and ruminative exploration

\begin{tabular}{lcccc}
\hline \hline & Estimate & Std. Error & $t$-value & $p$-value \\
\hline Intercept (Self-esteem) & 3.042 & .090 & 33.88 & $<.001$ \\
Exploration in-breadth & .285 & .023 & 12.39 & $<.001$ \\
Ruminative exploration & -.391 & .020 & -19.08 & $<.001$ \\
\hline
\end{tabular}

However, as hypothesized, exploration in-breadth was positively related to distress $(r=$ $.11, B=.16, p<.001)$. Despite higher endorsement of positive self-attitudes, participants also indicated higher frequency of negative emotions with an increase in future planning and exploration. Interestingly, deviating from our hypothesis, this relationship not only disappeared but reversed in direction after including ruminative exploration in the model. This indicated that the association with distress was indeed accounted for by ruminative exploration, which was positively associated with distress in the multiple regression model $(B=.61, p<.001)$. After partialling out the ruminative component, exploration in-breadth was negatively related to distress $(B=-.13, p<.001)$.

Table 5. Multiple regression results for distress with in-breadth and ruminative exploration

\begin{tabular}{ccccc}
\hline \hline & Estimate & Std. Error & $t$-value & $p$-value \\
\hline Intercept (Distress) & 1.075 & .150 & 7.160 & $<.001$ \\
Exploration in-breadth & -.131 & .039 & -3.397 & $<.001$ \\
Ruminative exploration & .612 & .034 & 17.848 & $<.001$ \\
\hline
\end{tabular}

\section{Discussions}

Results provided weak support for our hypotheses. In particular, as we predicted, there was a positive association between exploration in-depth and self-esteem that was not accounted for by the negative association between in-depth and ruminative exploration. This solidified the notion that a thoughtful identity exploration process may be related to adaptive outcomes; however, there was no negative association with distress. Similarly, although exploration inbreadth was positively related to self-esteem (contrary to our hypothesis), it was also positively related to distress. These results altogether highlighted the importance of including both 
measures of well-being and ill-being, because a positive relation with one does not necessitate a negative relation with the other. In other words, conclusions drawn about well-being can not be directly generalize to inference about ill-being, and vice versa.

An unpredicted yet intriguing finding was the association between exploration in-depth and distress only when ruminative exploration was included in the model. In other words, the portion of exploration in-depth that was unrelated to ruminative exploration was indeed predictive of low levels of distress. This is non-surprising in examining the bivariate correlation patterns: all exploration processes were positively related, despite including both adaptive and maladaptive constructs. As a result, in partialling out the maladaptive and ruminative component, a thorough investigation of one's future was indeed indicative of both well-being and a lack of ill-being. Further, the direction of the association between exploration in-breadth and distress completely reversed after including ruminative exploration in the model. Without the maladaptive component, there was now a negative association, once again highlighting the adaptive nature of the exploration process.

In examining the scale items that constituted the exploration variables, it became clear that exploration in-breadth did not fully capture the breadth of the exploration process in the same way that our theoretical arguments assumed. In particular, the hypothesized negative connotations of exploration in-breadth largely resulted from assuming a direction-less and superficial exploration process that cover pursuing various different identities without investigating any individual one closely. On the other hands, the scale items, such as "I think a lot about how I see my future" and "I try to find our which lifestyle would suit me" do not emphasize the existence of different options very directly. Thus, as a future direction, we should look more closely into the relationship between in-depth and in-breadth exploration to actually address the construct of interest: exploring multiple paths, each in a superficial manner.

The current paper provided a straightforward investigation of the relationships between different identity exploration processes and well-being as well as ill-being. In examining models with and without the ruminative term, we were able to directly investigate the correlates of adaptive identity processes. However, it is important to note the high collinearity among all current variables. As a future direction, we aim to use path analysis to more sensitively account for the cross-correlation among predictors and dependent variables across our different models. 
In this way, we would have more confidence about the outcomes and correlates of identity development, an essential normative process during emerging adulthood. 


\section{References}

Berzonsky, M. D. (1989). Identity Style: Conceptualization and Measurement. Journal of Adolescent Research, 4(3), 268-282. https://doi.org/10.1177/074355488943002

Crocetti, E., Rubini, M., \& Meeus, W. (2008). Capturing the dynamics of identity formation in various ethnic groups: Development and validation of a three-dimensional model. Journal of Adolescence, 31(2), 207-222. https://doi.org/10.1016/j.adolescence.2007.09.002

Erikson, E. H. (1968). Identity: Youth and Crisis. W. W. Norton \& Company.

Hemphill, J. F. (2003). Interpreting the magnitudes of correlation coefficients. American Psychologist, 58(1), 78-79. https://doi.org/10.1037/0003-066X.58.1.78

Kessler, R. C., Andrews, G., Colpe, L. J., Hiripi, E., Mroczek, D. K., Normand, S.-L., Walters, E. E., \& Zaslavsky, A. M. (2002). Short screening scales to monitor population prevalences and trends in non-specific psychological distress. Psychological Medicine, 32(6), 959-976.

Luyckx, K., Goossens, L., Soenens, B., \& Beyers, W. (2006). Unpacking commitment and exploration: Preliminary validation of an integrative model of late adolescent identity formation. Journal of Adolescence, 29(3), 361-378. https://doi.org/10.1016/j.adolescence.2005.03.008

Luyckx, K., Schwartz, S. J., Berzonsky, M. D., Soenens, B., Vansteenkiste, M., Smits, I., \& Goossens, L. (2008). Capturing ruminative exploration: Extending the four-dimensional model of identity formation in late adolescence. Journal of Research in Personality, 42(1), 58-82. https://doi.org/10.1016/j.jrp.2007.04.004

Luyckx, K., Soenens, B., \& Goossens, L. (2006). The personality-identity interplay in emerging adult women: Convergent findings from complementary analyses. European Journal of Personality, 20(3), 195-215. https://doi.org/10.1002/per.579 
Marcia, J. E. (1966). Development and validation of ego-identity status. Journal of Personality and Social Psychology, 3(5), 551-558. https://doi.org/10.1037/h0023281

Mastrotheodoros, S., \& Motti-Stefanidi, F. (2017). Dimensions of Identity Development Scale (DIDS): A test of longitudinal measurement invariance in Greek adolescents. European Journal of Developmental Psychology, 14(5), 605-617. https://doi.org/10.1080/17405629.2016.1241175

Meeus, W. (2011). The Study of Adolescent Identity Formation 2000-2010: A Review of Longitudinal Research. Journal of Research on Adolescence, 21(1), 75-94. https://doi.org/10.1111/j.1532-7795.2010.00716.x

R Core Team. (2020). R: A Language and Environment for Statistical Computing. R Foundation for Statistical Computing. https://www.R-project.org

Rosenberg, M. (1965). Rosenberg self-esteem scale (RSE). Acceptance and Commitment Therapy. Measures Package, 61(52), 18.

Rosenthal, D. A., Gurney, R. M., \& Moore, S. M. (1981). From trust on intimacy: A new inventory for examining Erikson's stages of psychosocial development. Journal of Youth and Adolescence, 10(6), 525-537. https://doi.org/10.1007/BF02087944

Schwartz, S. J., Beyers, W., Luyckx, K., Soenens, B., Zamboanga, B. L., Forthun, L. F., Hardy, S. A., Vazsonyi, A. T., Ham, L. S., Kim, S. Y., Whitbourne, S. K., \& Waterman, A. S. (2011). Examining the Light and Dark Sides of Emerging Adults' Identity: A Study of Identity Status Differences in Positive and Negative Psychosocial Functioning. Journal of Youth and Adolescence, 40(7), 839-859. https://doi.org/10.1007/s10964-010-9606-6 